\title{
Service Self-Organization Based on SON in LTE-Advanced Heterogeneous Networks
}

\author{
Gisung Lee ${ }^{1}$, Jongchan Lee ${ }^{2}$, Sangjoon Park ${ }^{2 *}$ and JinTae Jeong ${ }^{1}$ \\ ${ }^{1}$ Division of Computer \& Game, Howon University \\ ${ }^{2}$ Dept. of Computer Information Engineering, Kunsan National University
}

ygslee@howon.ac.kr

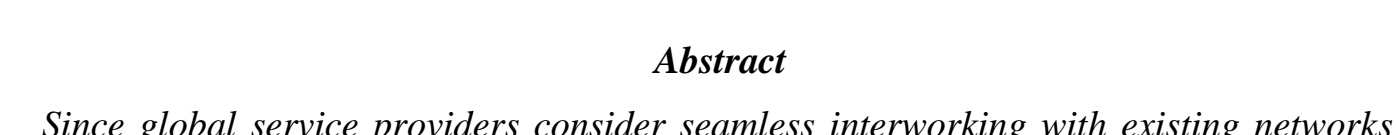

Since global service providers consider seamless interworking with xisking networks as the most important factor, it is most likely that LTE-Advanced of 3GPP is selected as international standard for $4 G$ wireless communi ation.' As sutch, we suggest SON based service self-organization which provides users with service continuty even when mobile users have terminal mobility and session mobility, and enable service providers to implement "heterogeneous multi-network of integrated" Which guarantees better service in terms of wireless communication access, transmission speed, and Quality of Service (QoS). It is also expected to allow fast handover servicesuch es KOD, and streaming in LTE-Advanced system. Moreover, this study could be conducted with various research institutions and manufacturers, leading joint research and technology transfer in the future.

Keywords: SON, Service Self-Organization, Service Continuity, Heterogeneous Networks

\section{Introduction}

For progressing to fourth-generation mobile communication, the need to evolve the systems towards supporting a) wider range of telecommunication applications, involving multimedia service, is increasingly gaining importance. According to this, ITU regulates $4 \mathrm{G}$ technique as IMT-Advanced, and defines high data rate, network convergence, ubiquitous and seamless connection as characteristics. As a technique which satisfies above mentioned conditions, LTE -Adyanced is in the spotlight now [1-3]. As shown in Figure 1, 3G LTE uses backbone based on ALL-IP, interworking with various networks seamlessly. The service offered from 3 GPP LTE-Advanced is always connected with data rate of over maximum $100 \mathrm{Mbp}$ and minimum delay; it is possible to seamlessly interwork with existing services in WCDMA and HSDPA networks. In case of selecting 3GPP LTE-Advanced, it is possible to link existing $3 \mathrm{G}$ and $4 \mathrm{G}$ networks, so all of communication functions could be used with only one mobile terminal. The most remarkable technique for this is service continuity technique through seamless connection [4-8].

LTE-Advanced should provide high-speed data communication mainly in hot-spot area, and offer service continuity during terminal mobility and session mobility with only a mobile terminal. However, in LTE-Advanced where different networks coexist, it is actually difficult to provide service continuity efficiently [9-11]. And because each resource in LTE-Advanced system and the network state such as bandwidth, error rate change variably, it is considered

* Corresponding author: SangJoon Park 
that it is impossible to control service continuity with procedural, artificial, and static control method applied to the existing voice service.

As a solution for this, we introduce a self-organizing network (SON) concept in LTEAdvanced. it is aimed at making heterogeneous networks expand more stably and efficiently through applying self-organization concept that different networks unite and interact for better effect, so that it has received a lot of attention as core technology to apply commercial system of future mobile communication networks after fourth-generation as well as LTEAdvanced [11-13]. As of yet, it mostly focuses on progress of physical aspect such as setting up base stations, performance improvement and capacity expansion, but it is thought that if a method which guarantees service continuity based on self-monitoring technique is developed, it will play a key role in market enlargement of LTE-Advanced; self-monitoring is a technology for performing traffic control and improving resource capacity byanalyzing some context information about neighboring cells and terminals, which is the core technology of SON [14-15].

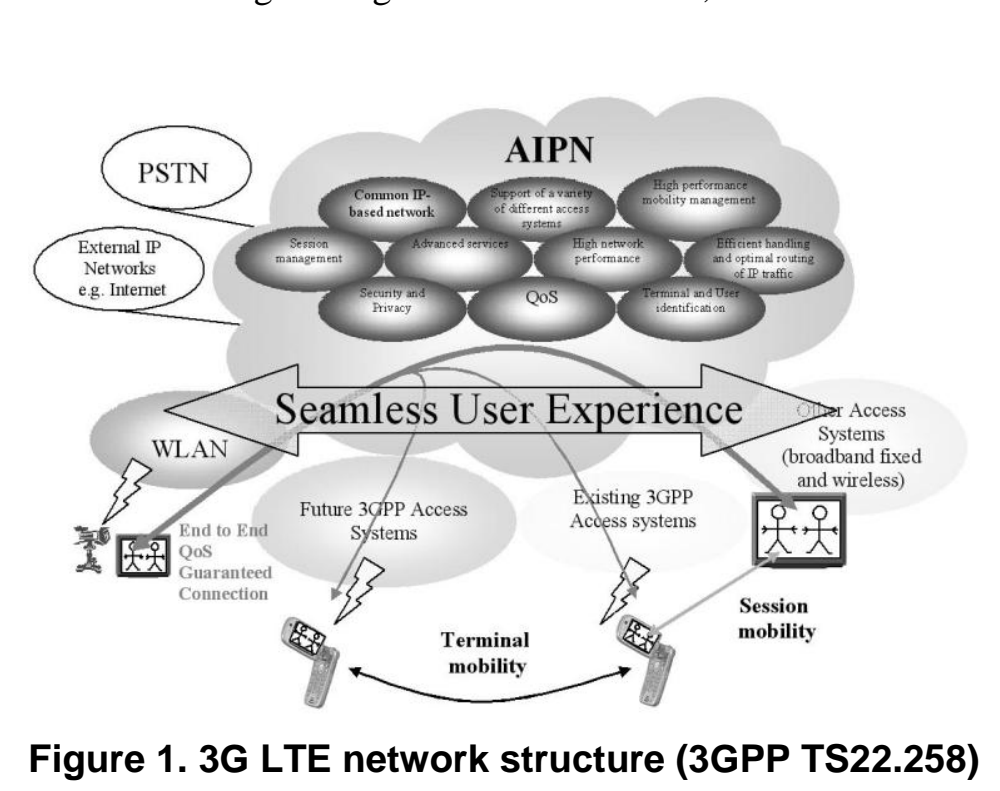

We propose a service self-organization mechanism to support the service continuity effectively based on SON, in which a mobile terminal collects some information about its current state of each cell and a base station, based on the information collected by monitoring inner or adjacentcells, shares related data and converges, controlling service continuity on its own. In other words, as context information of mobile terminals and cells changes, the operation of related functions such as ISHO, cell selection, source allocation, load control, and OOS mapping is adapted; each function fits into the change, exchanges the process of reorganization, and interacts; these actions go toward to satisfy service continuity.

\section{Previous Works}

3GPP is an organization for standardizing LTE which is called 3.9th generation with GSM, WCDMA, HSDPA used by current $89 \%$ of mobile communication subscribers. It has been conducting the standardization of LTE-Advanced for satisfying the system requirement of IMT-Advanced through complement of LTE technology. Although it is hard to expect that LTE-Advanced is more progressed in epochal technology than LTE, it is supposed to be improved in the field of broadband support, multiple antenna expansion, network cooperative communication, SON technology. The study on the concept of common radio resource 
management, whose objective is to manage each access network synthetically to supply ISHO function and to optimize the performance in various heterogeneous access networks, is being carried on actively. Especially, 3GPP suggests a network structure for integrated radio resources management in TR25.881 the technology standard text and common radio resource management (CRRM) the method of the integrated radio resources management to support that, and many projects to modify and complement the weak point of CRRM are being proposed.

SON is aimed to organize the network stable, efficient and scalable using the concept of self-organization. Especially, with introduction of femto cell and next generation 4G visualized in mobile communication system, SON which includes the automated/functions such as self-organization or self-configuration is estimated as a key technology of commercialization. It is because SON technology, which is able to realize the simplification of management, convenience and reduction of operation and support cost, fncrease of capacity, and so on, is considered as an optimum alternative with reduction of total cost of ownership and improvement of managerial efficiency emerged as key words of service providers. Especially, each major equipment company also develops SON technology and devotes all their efforts to secure service providers which need to curtail the cost due to explosive increase in the amount of using data and a flat rate system. Because the sections which self-organization can be applied are realfy yarious in the mobile communication field, the concept of self-organization can be usêd for the indiviđualmanagement algorithm and the optimization technique according to the characteristres and types of network which is intended to organize in self-organization

\section{Proposed Scheme}

If LTE-Advanced becomes) 4G-integrated standard, and a commercialization, service providers which have various access netvorks, could combine a variety of access networks based on IP core network without another investment for networks. This paper, with this industrial background, suggests a fyered mechanism for supporting service continuity using SON, which is core part for commercial system application in LTE-advanced heterogeneous networks. Figure 2 shous (a) two-layered control mechanism for the service of selforganization based on SON

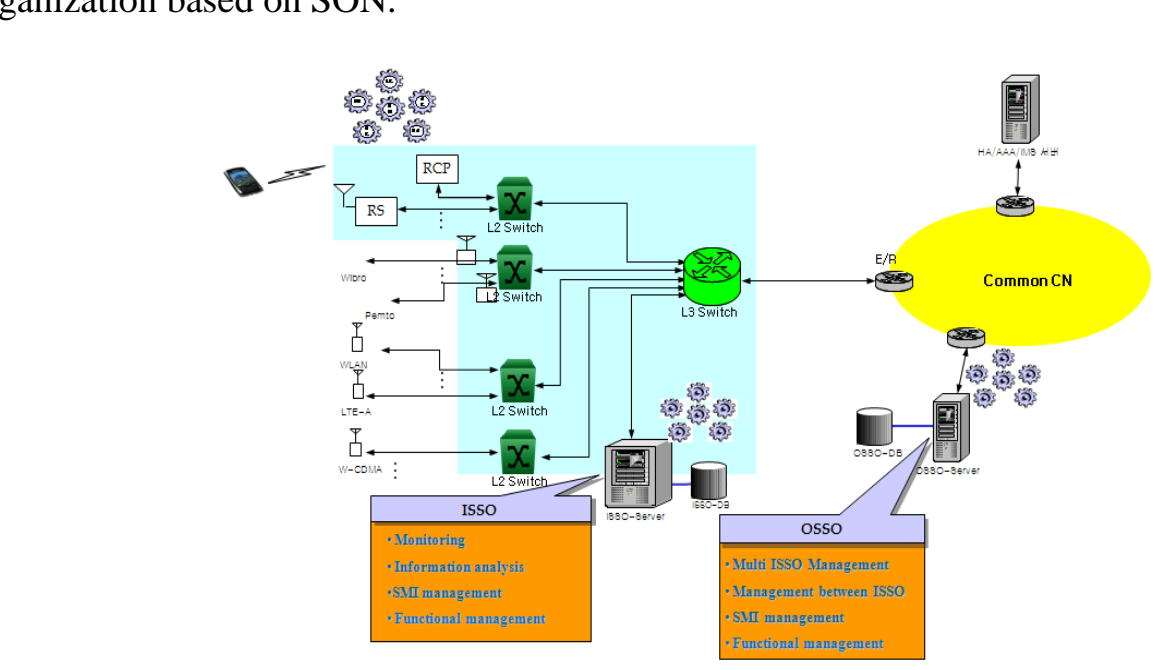

Figure 2. Two-layered control mechanism for service self-organization 
Previously, to offer ISHO and related functions in various heterogeneous wireless networks, the researches on the integrated radio resources management which could manage each access network resource synthetically and optimize whole system operation, has been processed; there is CRRM of TRS25.88 as a representative method. However, CRRM, by focusing only on controlling sub-access networks to integration and distribution with existing $\mathrm{RM}$, had a fatal defect that interworking mechanism between functions and related entities lack to support service continuity required in 4G. Especially, in LTE-Advanced which promotes the commercial service after 2015, it is obvious that convergence and interworking among functions according to use case are necessary to satisfy service requirements such as end-to-end QoS \& guaranteed connection. Accordingly, the goal of this research is to propose the supporting mechanism which makes service continuity possible by enabling functions related to the state change of mobile terminal and base station to make selr-control/ adaptation/ reconstruction through the suggested service self-organization.

\subsection{SON based service control mechanism}

The service of self-organization, which applies SON-based/contro/ mechanism different from CRRM (TR25.881), is the overlaid control mechanism based on distributional control by two-layered structure to be supportable for both tightly coupling and loosely coupling network able to be organized according to service proxiden's network configuration. As shown in Figure 3, the inner-service of self-organization (ISSO) is located in local networks to support service continuity for seamless mobility occulring between tightly coupling subnetworks in LTE-Advanced efficiently; the onter-service of self-organization (OSSO) is located in external network to rinanage the majority of ISSO and to support service continuity between loosely coupling sub-heterogeneous netyorks.

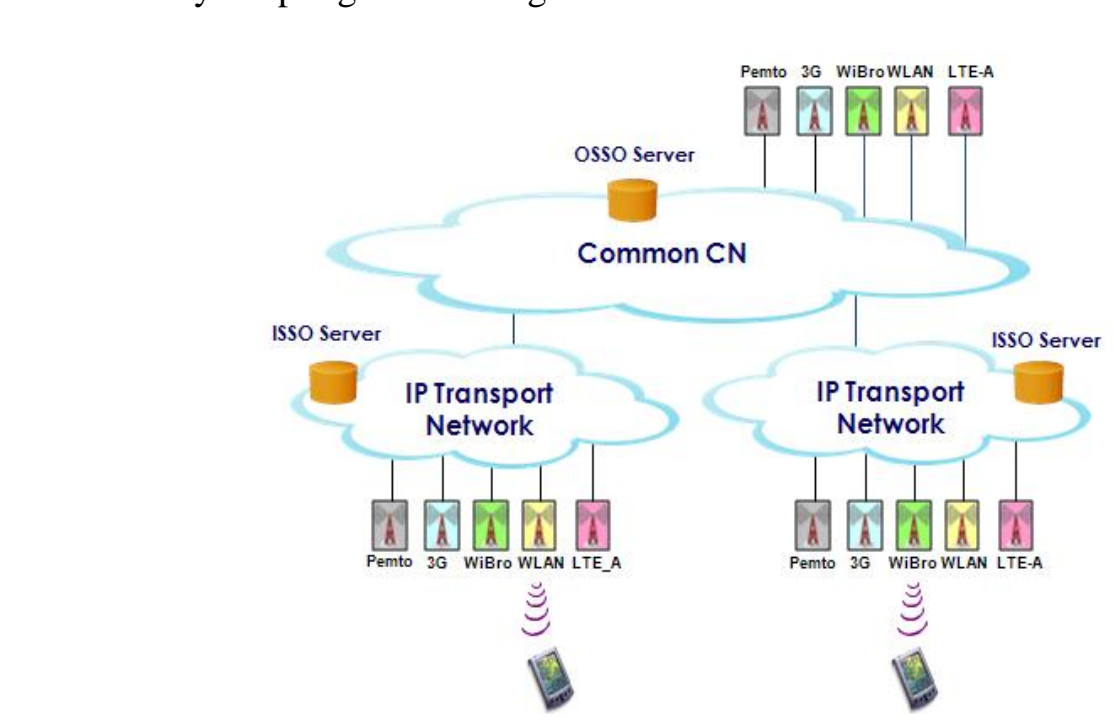

Figure 3. Two-layered control mechanism for service self-organization

Figure 4 shows the proposed control hierarchical control structure to support the service of self-organization in LTE-Advanced. All functions are located at ISSO of LTE-Advanced access network and OSSO of LTE-Advanced core network; each function is as follows; Service Management Index (SMI) rule maker which describes SMI of service providers, SMI monitor which collects the conditions applying to SMI, and SMI firer which handles functions the control system should conduct directly by Lego method. 


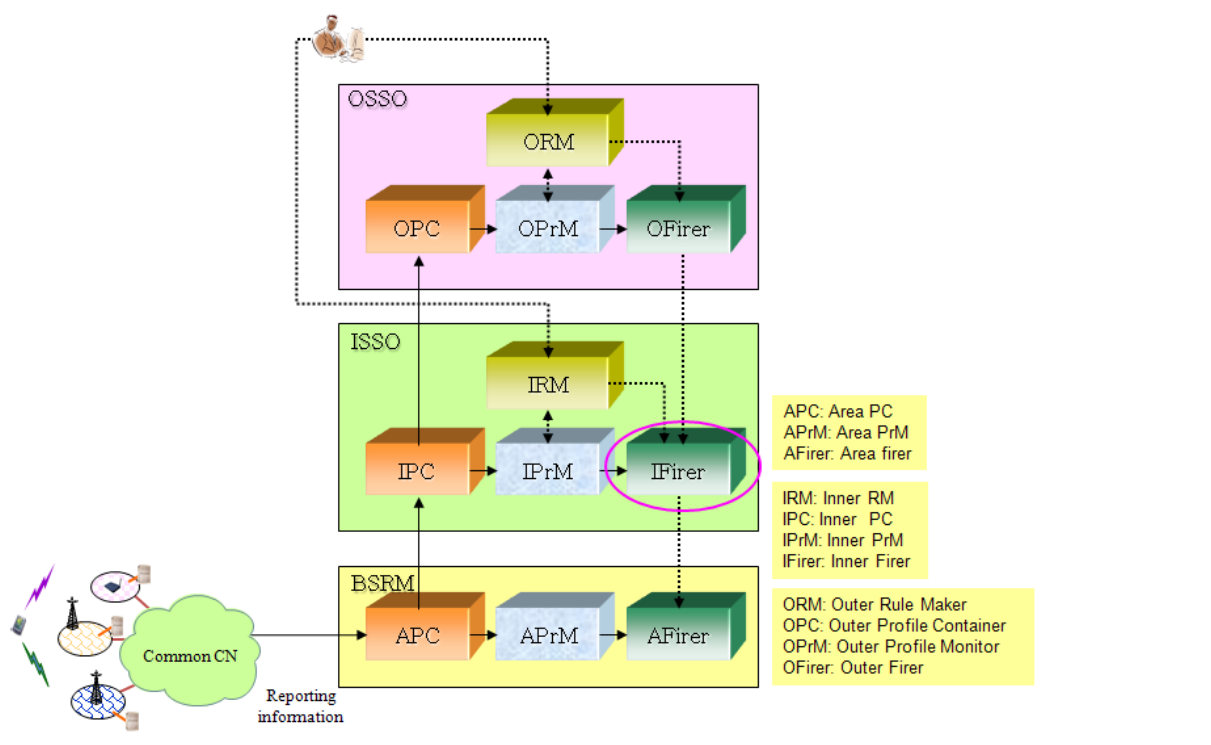

Figure 4. Hierarchical structure for seryice self-organization

ISSO/OSSO is designed so that both tightly and loosely coupling network can be supportable, and the service of self-organization mechanism is developed based on regional distribution and integration controf of ISSO/OSSO. For this, ISSO is developed to be capable of deletion of unnecessary function and addition of function by implementing the embedded module type interconnecting with protocol.

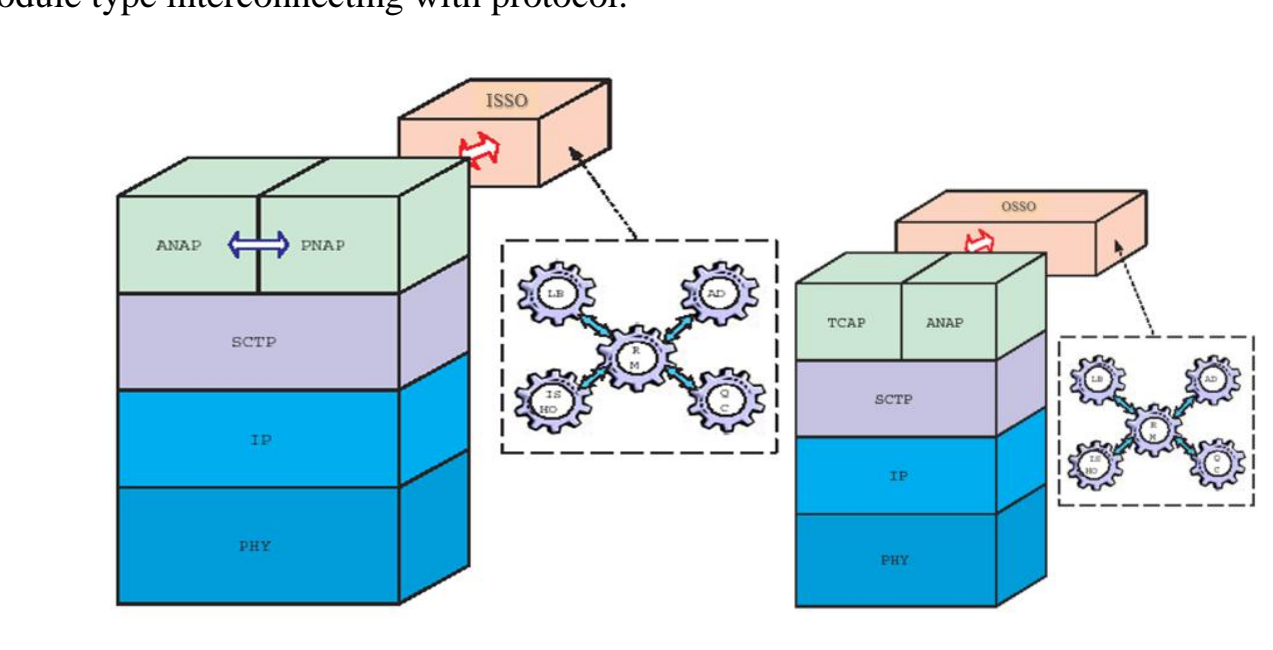

Figure 5. Interworking structure for service self-organization

\subsection{Gearing method}

To adapt to the dynamically changing environment around mobile terminals and cells, it should be capable to make self-control/adaptation/reconstruction of functions. As shown in Figure 6, the control interface based on Gearing is designed so that each function for service self-organization can be combined according to use case. After each algorism is designed as module to be able to conduct independently in real-time processing of service continuity, information sharing and dynamic adaptation are composed for all functions to share and 
adjust the information in order to exclude the confused connection in the coordination between the functions and to exclude the reason of the bottleneck. For this, the service of selfadaptation method and the service of self-monitoring method are considered. The service of self-monitoring analyzes the current state based on internal information and external information collected through monitoring mobile terminals and cells. The functions to operate in each use case are selected from the result of circumstantial judgment. The service of selfadaptation is conducted to control the related parameter automatically to be suitable for conditions. Each function with operation mechanisms interacts, reciprocating the process to reconstruct and adjust to changes.

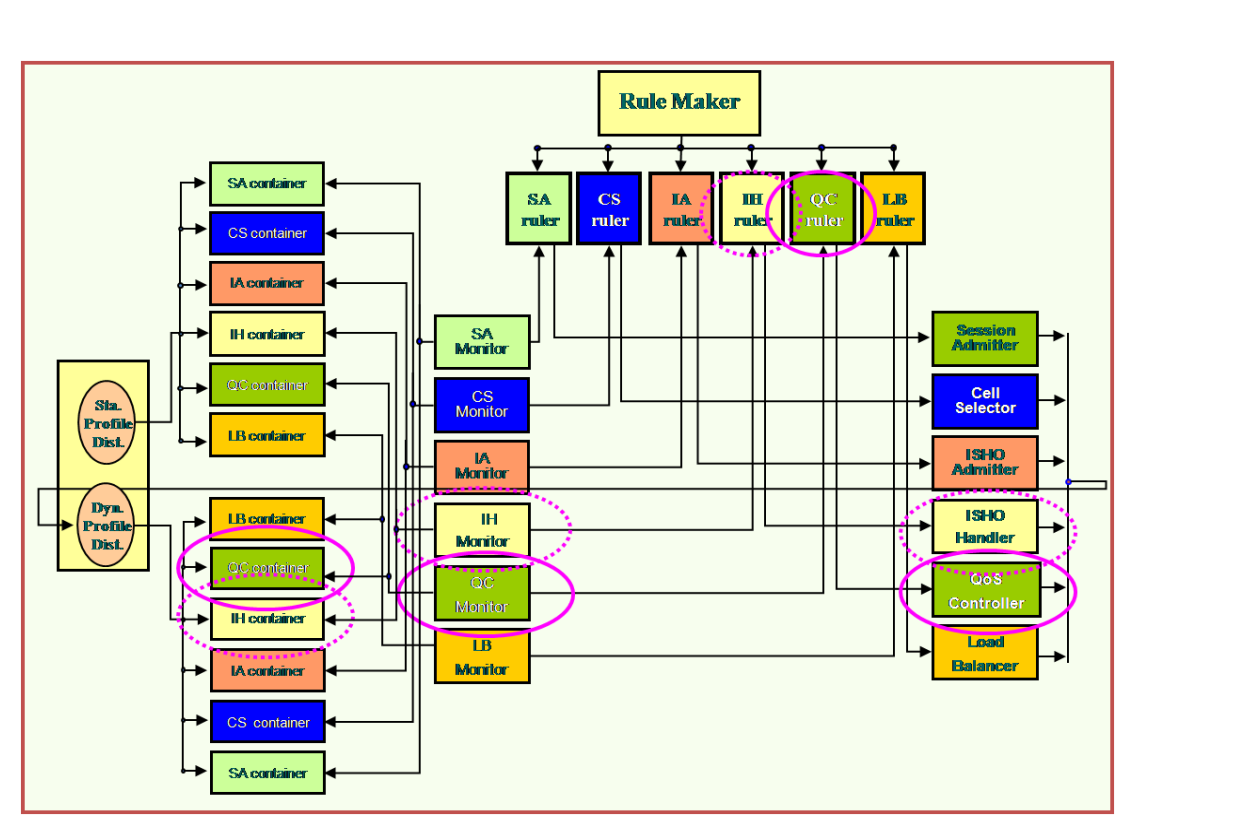

Figure 6. Service self-organization mechanism based on Gearing method

The procedure which the service of self-organization is adapted is as following. All containers for monitoring in networks collect reporting information such as SLA information, accessible cell inforiation of each mobile terminal, and QoS of mobile terminals; Monitor is periodically implemented by the Condition of Firing SMI (CFS). If one condition of the CFS is satisfied, Conditioner sends a message to SMI Ruler for ruling. SMI Rule maker decides what function to conduct through already established SMI, and delivers Firing message including SMI information to related Firer, which could operate suitably for SMI through it. Each functional algorism getting firing messages conducts actions for SMI decision and sends the result to Sub-Firer. Each rule is located separately in ISSO and OSSO according to its role. These procedures can be conducted in the process of service operation, and adapted on the way of transmission of traffic after completion of service operation.

\subsection{Lego method}

In order to effectively implement the service of self-organization and commercialize it, coping adaptively with the management direction of each service providers, Lego based function combination is considered. Through this, open interface, modularity, flexibility, commonality can be satisfied at the same time. This applies the modularization scheme used in mechanical engineering, and is an epochal lego-style treating method which enables 
adaptive combination and separation of different functions by stratifying and dichotomizing interface with lego-style adapted between the functions. Figure 7 shows an example of the combinable function according to use case. For example, despite the new session requirement, functions to use are selected by conditions of firing SMI and monitoring some information such as user, terminal, near base station, and radio condition. The optimum result in current condition can be obtained by making different parameters between the shared functions.

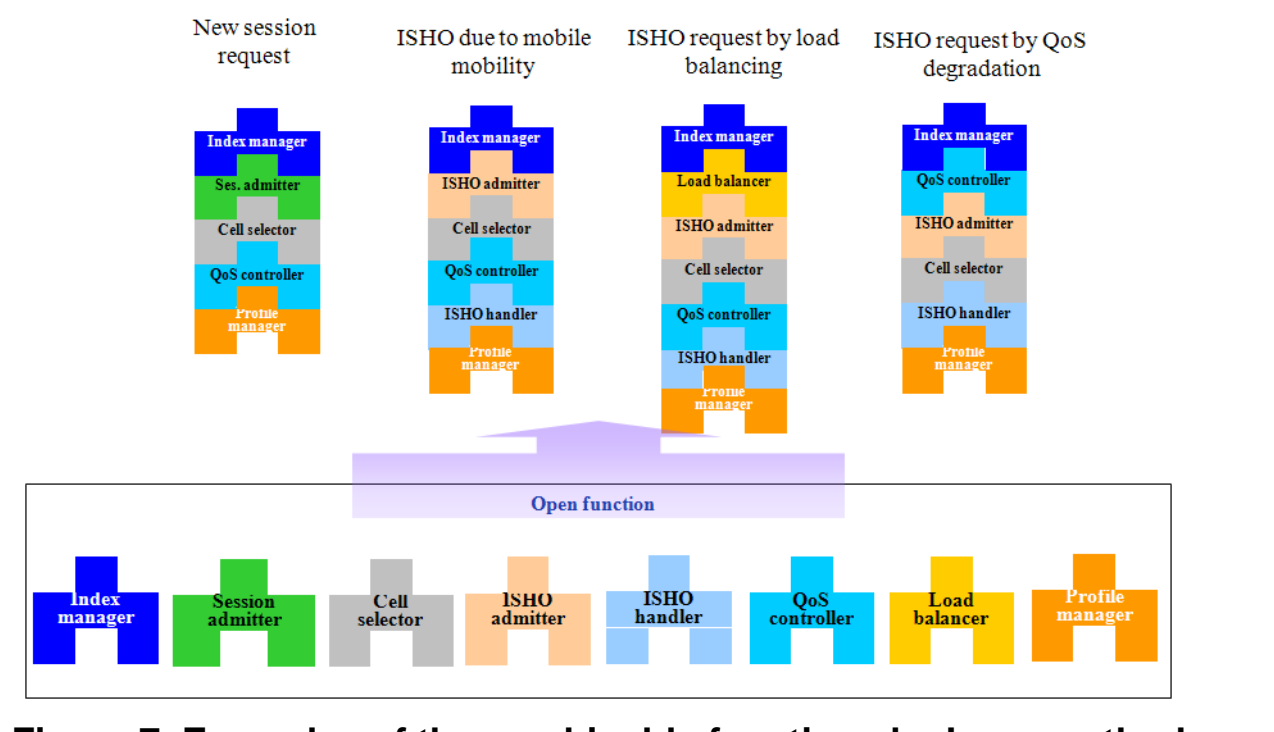

Figure 7. Examples of the combinable functions by Lego method

Lego based function combination is designed for acting in each parameter appropriate for service area where mobile terminals are located, by deciding action of each function and optimizing separately only with locan nformation according to distributed processing concept of the service of self-organization. For this, Lego based function combination needs three element technologies from the technical point of view. Those three element technologies are as follows; dynamically interconnecting scheduler which combines functions according to use case putting lego based function combination to use; interconnecting structure by function which extracts composition elements of each function, i.e. terminal elements, system elements including base station, wireless network element, and formulates their correlation according to use-case; interconnecting structure by parameter for efficient adaptation of related parameter according to environmental change; task-based design for optimizing operation as well as minimizing behavior process. Figure 8 shows interconnecting structure for allocation of shared value during cell selection, in case of terminal mobility.

\begin{tabular}{|l|l|}
\hline Title & MISHOCellSelection \\
Subject & ISSO \\
Initiator & MT \\
Target & MT \\
On & MT $==$ Mobility \\
Value & If \\
& (ISHO == MISHO) \\
& Then \\
& (Cell Average Delay $::=$ MTI) \\
& \&\& \\
& (Packet Loss $::=$ I) \\
& $\& \&$ \\
\hline
\end{tabular}




\begin{tabular}{|l|l|}
\hline & $($ Mobile Speed $::=\mathrm{I})$ \\
$\& \&$ \\
$($ Cell Load $::=\mathrm{LTI})$ \\
$\& \&$ \\
(Maximum Bit Rate $::=\mathrm{MEI})$ \\
\\
\end{tabular}

\section{Figure 8. An example of the combinable function for cell selection}

\section{Conclusion}

The proposed service scheme of self-organization is very important ractor that could affect the performance of the integrated system. Also IPR is also/expected to become important. Initial success of LTE-Advanced related technology developed based on this scheme would give us an advantageous position for developing other related technologies - standardizing them, and preempting emerging technology. Accordingly, we would be able to respond promptly to the trend in international research and patênt application by collecting all the related technologies required for the serviee continuity in LTE-Advanced system and establishing source technology based opthem.

\section{Acknowledgements}

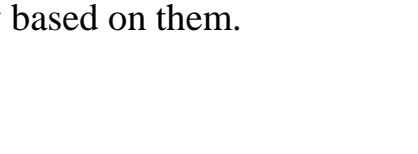

This work was supported by the Howon University Fund. 2013.

\section{References}

[1] B. Wang, et a., "Primary-Prioritzed Markov Approach for Dynamic Spectrum Allocation”, IEEE Trans. Wireless Communjcation, vol \& no. 4, (2009) April, pp. 1854-1865.

[2] S. H. Ali, K. D. Lee and V. C.M. Leung, "Dynamic Resource Allocation in OFDMA Wireless Metropolitan Area Networks", IEEE Wifeless Communications, vol. 14, no. 1, (2007) February, pp. 6-13.

[3] P. Calduwel Newton and 4 . Arockiam, "An Intelligent Technique to Improve Quality of Service (QoS) in Multihomed Mobile-Networks", International Journal of Advanced Science and Technology, vol. 7, (2009) June, pp. 11-20.

[4] IEEE Std 802.16m-2011, "Part 16: Air Interface for Broadband Wireless Access Systems", Amendment 3: Advanced An Tnterface, (2011) May.

[5] A. A. Abbasi and M. Hussain, "A QoS Enhancement Framework for Ubiquitous Network Environments", International Journal of Advanced Science and Technology, vol. 43, (2012) June, pp. 37-48.

[6] 5. Buljore, et al., "Architecture and Enablers for Optimized Radio Resource Usage in Heterogeneous Wireless Access Networks: The IEEE 1900.4 Working Group", IEEE Commun. Mag., vol. 47, no. 1, (2009) January, pp. 122-129.

[7] X. Zhou, et al., "Traffic-Driven Dynamic Spectrum Auctions", Sensor, Mesh and Ad Hoc Communications and Networks Workshops (SENCON), (2008).

[8] W. G. Chung, et al., "Calculation of Spectral Efficiency for Estimating Spectrum Requirements of IMTAdvanced in Korean Mobile Communication Environments", ETRI J., vol. 29, no. 2, (2007) April, pp. 153161.

[9] H. -J. Shin, M. -G. Kang and K. -H. Choi, "Design, Modeling, and Performance Analysis of Web-based Multi-purpose Discovery Middleware in Ubiquitous Environments", International Journal of Multimedia and Ubiquitous Engineering, vol. 7, no. 3, (2012) July, pp. 1-10.

[10] A. Kakhbod and D. Teneketzis, "Power allocation and spectrum sharing in multi-user, multi-channel systems with strategic users", IEEE Transactions on .Automatic Control, no. 99, (2012).

[11] A. P. Subramanian, et al., "Near-Optimal Dynamic Spectrum Allocation in Cellular Networks", New Frontiers in DynamicSpectrum Access Networks (DySPAN), (2008) October. 
[12] K. Ayyappan and R. Kumar, "QoS Based Vertical Handoff Scheme for Heterogeneous Wireless Networks", International Journal of Research and Reviews in Computer Science (IJRRCS), vol. 1, no. 1, (2010), pp. 1-6.

[13] M. R. Lee and T. T. Chen, "Trends in Ubiquitous Multimedia Computing", International Journal of Multimedia and Ubiquitous Engineering, vol. 4, no. 2, (2009) April, pp. 115-124.

[14] 3GPP TSG RAN WG1, R1-0911270, "Relay Frame structure and design of TDD made", Huawei, (2009) March.

[15] Q. Liu, N. Linge and F. Gao, "A Context-aware Protocol for Location Discovery Exchange in a Ubiquitous Environment", International Journal of Multimedia and Ubiquitous Engineering, vol. 7, no. 2, (2012) April, pp. 177-182.

\section{Authors}

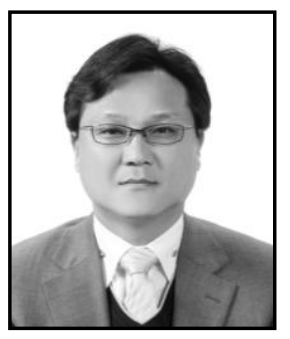

\section{GiSung Lee}

August, 2000: A Ph.D degrees in computer science and engineering from Soongsil University, Korea

From 2000 to 2005: a senior member of engineering staff in Electronics and Telecommunications Research Institute (ETRI)

The present: An associate professor in the Department of Computer Information Engineering, Kunsan National University

Major area of interest. Mrobile Multimedia, Forensic, System Security

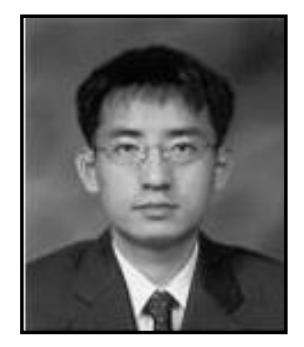

\section{JongChan Lee}

August, 2000: A Ph.D degrees in computer science and engineering from Soongsil University, Korea

From 2000 to 2005: a senior member of engineering staff in Electronics and Telecommunications Research Institute (ETRI)

The present: An associate professor in the Department of Computer Information Engineering, Kunsan National University

Major area of interest: Mobile Multimedia, Forensic, System

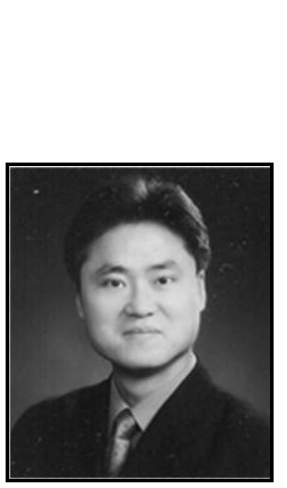

Security.

\section{SangJoon Park}

August, 2002: A Ph.D degrees in computer science and engineering from Soongsil University, Korea

From 2004 to 2007: Research Professor, Information \& Media Technique Institute, Soongsil University, Korea.

From 2007 to the present: An associate professor in the Department of Computer Information Engineering, Kunsan National University

Major area of interest: Mobile Multimedia, Simulation \& Modeling, System Security. 
International Journal of Multimedia and Ubiquitous Engineering Vol.9, No.1 (2014)

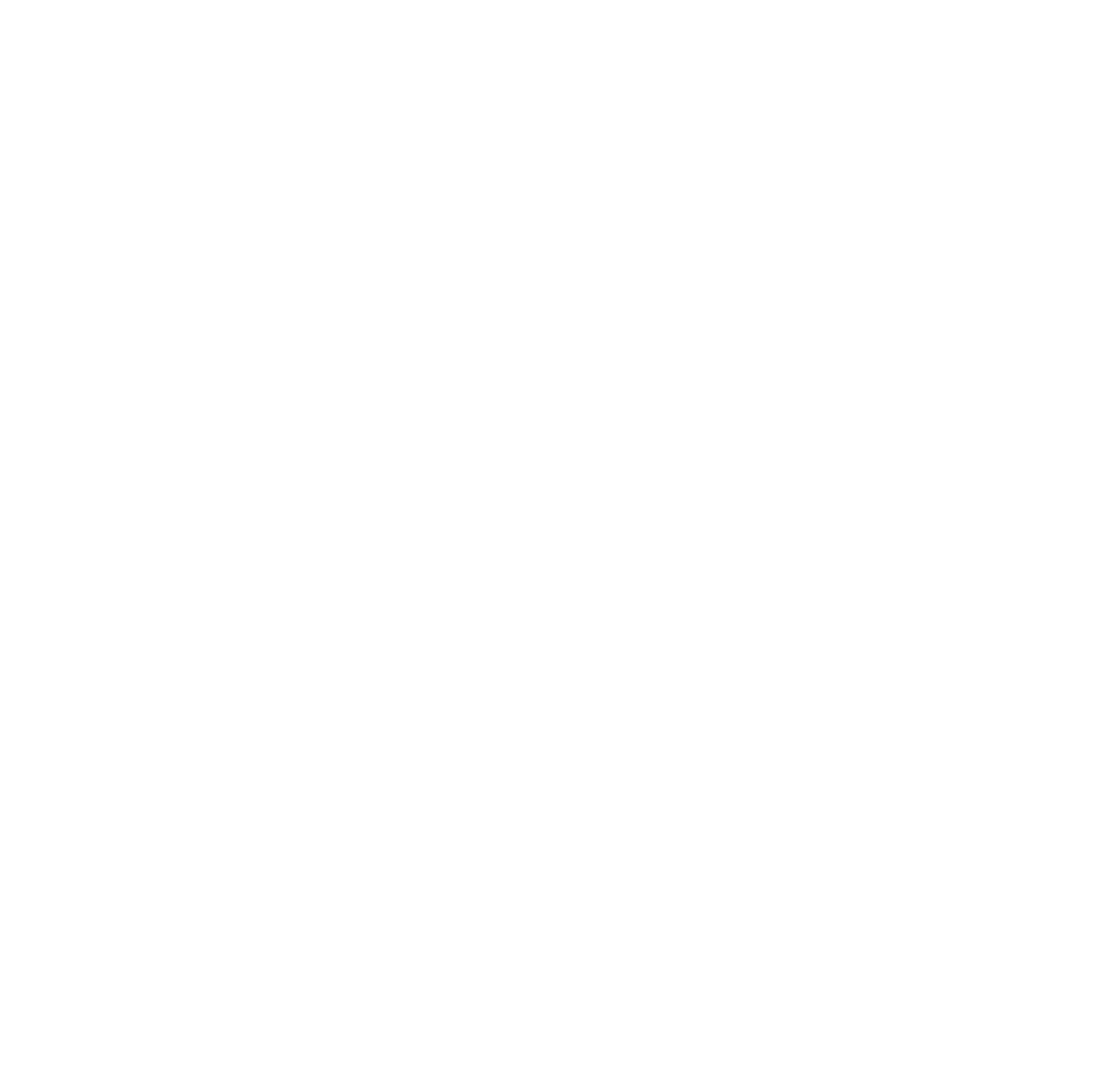

